\title{
La domestication des poissons du Mékong : les enjeux et le potentiel aquacole
}

Philippe Cacot

Jérôme Lazard

Cirad

Département Persyst

UPR «Aquaculture et gestion des ressources aquatiques $»$

TA B-20/01

Avenue Agropolis

34398 Montpellier cedex 05

France

<phcacot@aol.com>

<jerome.lazard@cirad.fr>

\begin{abstract}
Résumé
La pisciculture dans le bassin du Mékong repose, pour une bonne part, sur la production d'espèces indigènes. La domestication est une pratique traditionnelle dans le prolongement de la pêche. Aujourd'hui, 32 espèces indigènes sont produites à des niveaux divers; le poisson-chat, Pangasianodon hypophthalmus, domine largement avec plus d'un million de tonnes, suivi par trois espèces avec plusieurs dizaines de milliers de tonnes produites pour chacune ; il s'agit du silver barb (Barbodes gonionotus), du snakehead fish (Channa micropeltes) et de la climbing perch (Anabas testudineus). Elles sont bien adaptées aux conditions d'élevage de la région, étangs, cages et rizipisciculture, et elles peuvent être produites à différents niveaux d'intensification. Le rendement en étangs est généralement élevé avec les espèces qui ont une respiration aérienne complémentaire (jusqu'à $1000 \mathrm{t} / \mathrm{ha}$ par an de $P$. hypophthalmus en enclos dans le Mékong). La disponibilité en alevins constitue le principal facteur déterminant dans le choix des espèces, suivi par la rusticité et la valeur marchande. Le régime alimentaire intervient peu, mais ce paramètre va devenir important compte tenu de la raréfaction du poisson fourrage utilisé traditionnellement. Par ailleurs, le contexte évolue rapidement avec la diminution des stocks de populations naturelles de poissons, l'introduction d'espèces exotiques et la production d'hybrides pour la pisciculture. La domestication devient alors un moyen privilégié de préserver la biodiversité ; les programmes de R\&D en cours concernent surtout des espèces menacées.
\end{abstract}

Mots clés : domestication ; espèce indigène ; Mékong ; modèle ; pisciculture.

Thèmes : productions animales; pêche et aquaculture ; ressources naturelles et environnement.

\section{Abstract \\ Domestication of the indigenous Mekong fish species: The issues and the aquaculture potential}

Indigenous fish species are widely cultured in the Mekong River basin. Domestication is a traditional practice originating from fishing activity. Nowadays, 32 native species are produced at various levels. The Mekong catfish Pangasianodon hypophthalmus is the main species cultured with a production level of more than one million tons per year (2007). Several tens of thousands of tons are produced from each of the three other major species: the silver barb (Barbodes gonionotus), the snakehead fish (Channa micropeltes) and the climbing perch (Anabas testudineus). All these species fit into the local fish culture systems based either on the use of ponds, cages or rice fields. The production systems range from extensive to very intensive conditions, with highest yields obtained with the air breather species (up to 1,000 tons/ha/year of $P$. hypophthalmus in pens in the Mekong River). For a given species, the availability of fish seeds is the main constraint, followed by hardiness and market value. Surprisingly, the feeding regime is a minor factor but it will most probably soon become crucial due to the forecasted shortage of trash fish commonly used as fish feed. Moreover, the Mekong environmental conditions are changing quickly with the depletion of wild fish stocks, the introduction of exotic species and the production of hybrids for aquaculture. Domestication is thus becoming a means of protecting biodiversity. The ongoing R\&D programmes are therefore mainly focusing on endangered species.

Key words: domestication; fish culture; indigenous species; Mekong; models.

Subjects: animal productions; fishing and aquaculture; natural resources and environment. 
e bassin du fleuve Mékong (figure 1) est un foyer actif de domestication des poissons pour la pisciculture; une trentaine d'espèces indigènes font l'objet d'élevage à des degrés divers. L'une de ces espèces, le poisson-chat Pangasiidae, Pangasianodon hypophthalmus, est devenue, en une dizaine d'années, un des poissons majeurs de l'aquaculture mondiale avec un million de tonnes produites dans le seul delta du Mékong au sud du Vietnam (source : statistiques de l'Institut de recherche en aquaculture du Vietnam). Plusieurs autres espèces présentent un potentiel remarquable et diversement valorisé aujourd'hui. La domestication des poissons du Mékong profite d'une diversité remarquable avec 600 espèces peuplant le Bas-Mékong (Rainboth, 1996) et d'une longue tradition de pêche et d'aquaculture (Chevey et Le Poulain, 1940 ; Cacot, 1993) (figure 2).

La diversité ichtyologique est liée à la nature du bassin-versant, ainsi qu'à son histoire géobiologique. L'actuel bassin du Mékong résulte, en effet, de la fusion de plusieurs bassins fluviaux qui ont apporté des espèces différentes. Par ailleurs, les conditions hydrologiques du fleuve sont extrêmement contrastées entre la saison sèche et la saison des pluies; en période de crue, le débit du fleuve est considérable (le troisième débit maximal au monde après l'Amazone et le Brahmapoutre, d'après Welcomme (1985), et il est 30 fois plus élevé qu'en période d'étiage (statistiques du Mekong River Commission [MRC]) ; c'est l'une des plus fortes amplitudes connues. Une vaste plaine d'inondation se forme chaque année, de juillet à octobre en particulier, dans le bassin du grand lac Ton Le Sap au Cambodge et dans le delta au Vietnam ; les zones humides du fleuve couvrent au total $83700 \mathrm{~km}^{2}$. Les poissons locaux présentent des adaptations particulières à ce rythme hydrologique marqué ; celles-ci sont mises à profit par la pisciculture pour se développer dans des conditions spécifiques.

La domestication des poissons du Mékong est présentée, ici, au niveau de son évolution historique et récente. Nous essayons également de décrypter le potentiel aquacole des espèces, notamment, pour comprendre le niveau de leur production actuelle. Enfin, nous présentons brièvement l'adéquation des espèces aux différents systèmes de productions de la région. Cette présentation est le fruit de travaux de recherche et d'études sur l'aquaculture de cette région, menés par le Cirad depuis 1994.

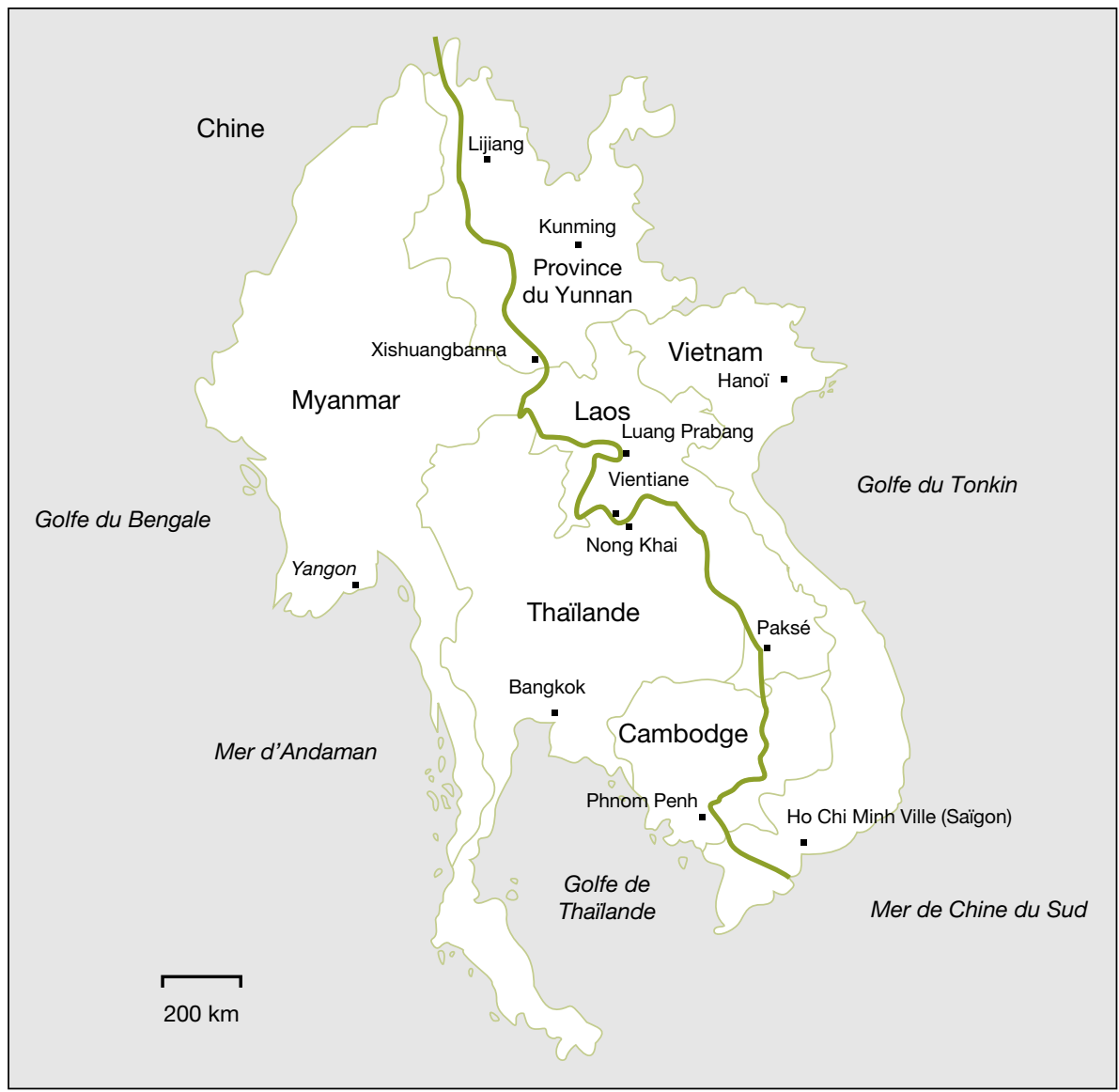

Figure 1. Carte du Bas-Mékong (d'après Baran, 2005).

Figure 1. Map of the lower Mekong river basin (from Baran, 2005).

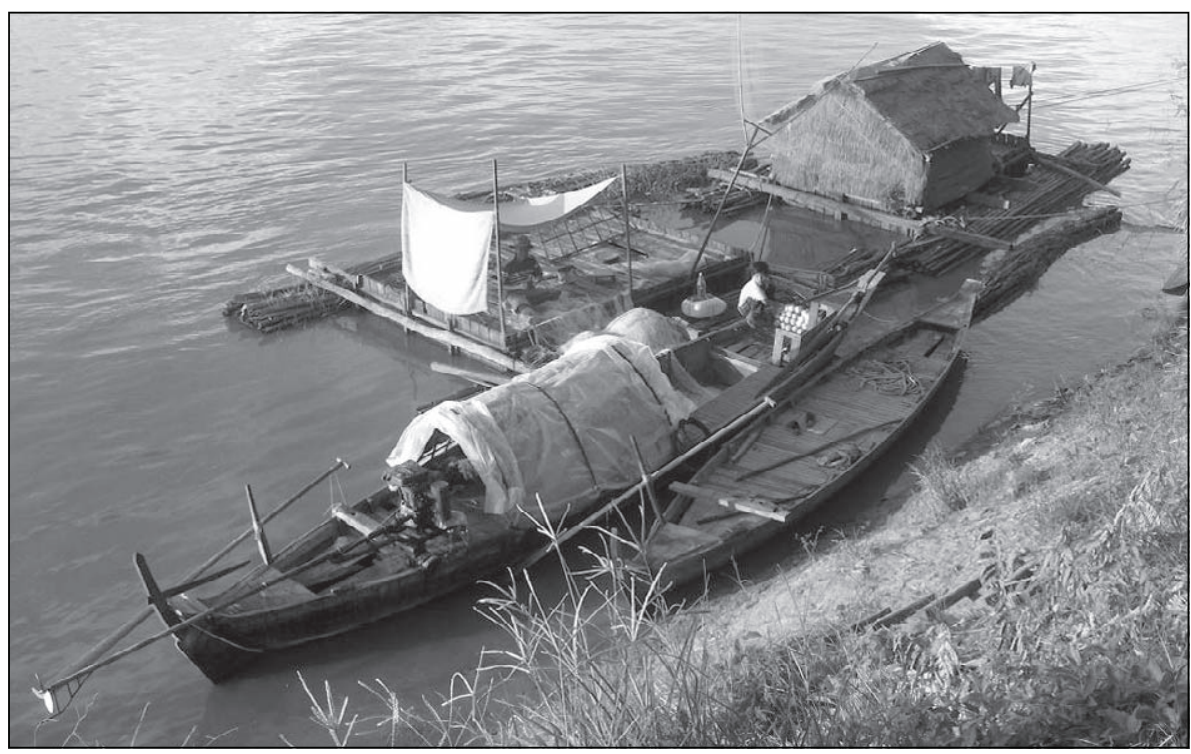

Figure 2. Pêcheur-pisciculteur au Cambodge.

Figure 2. Fisherman and fish farmer in Cambodia.

Les poissons sont élevés en cages flottantes et l'habitation est également flottante. 


\section{Un peu d’histoire}

\section{À l'origine}

La domestication des poissons du Mékong s'inscrit dans un continuum historique. Les populations riveraines du fleuve ont toujours consommé les poissons locaux, et tout particulièrement les poissons d'eau douce; ceux-ci couvrent jusqu'à la moitié des besoins en protéines au Laos. La relative abondance des poissons se caractérise, toutefois, par une saisonnalité marquée de la pêche, en fonction de la crue et de la décrue et des migrations de poissons qui sont associées. C'est dans ce contexte qu'est, naturellement, apparue l'aquaculture dans le bassin inférieur du Mékong.

Les formes " ancestrales " de pisciculture ont longtemps tiré profit de l'alternance des périodes d'abondance et de raréfaction des poissons. Elles reposent toutes sur la collecte de poissons juvéniles dans le milieu naturel, durant une période de relative abondance en début de saison des pluies, et la récolte des poissons de pisciculture en saison sèche. C'est le cas en particulier des snakehead fish (Channa sp.), très prisés pour l'élevage en cages, des poissons-chats Pangasiidae élevés en cages ou en étangs et des cyprinidés élevés préférentiellement en étangs ; c'est aussi le cas de plusieurs petites espèces qui colonisent les rizières durant la crue. On dénombre, ainsi, déjà une dizaine d'espèces exploitées dans ces systèmes traditionnels les plus anciens. L'alimentation de ces poissons a longtemps reposé sur le poisson dit "fourrage ", capturé localement, et le son de riz, également produit localement. La pratique de la riziculture irriguée, en apportant l'eau et le son de riz, constitue un élément clef du développement de l'aquaculture dans le bassin du Mékong, tout comme partout ailleurs en Asie.

\section{Évolution ancienne}

Les systèmes traditionnels ont progressivement évolué pour optimiser la production au gré des contraintes comme la disponibilité des structures d'élevage (étangs, cages), l'accès au marché. Le choix précis des espèces en est une bonne illustration. Ainsi, parmi les espèces de snakehead fish, Channa micropeltes est la préférée des pisciculteurs de par sa croissance, la plus rapide. Parmi les pangasidés, $P$. hypophthalmus et Pangasius bocourti sont préférés en étangs et en cages, respectivement du fait de leur adaptation optimale au milieu d'élevage (Cacot, 1993; Guibert, 2003; Huillery 2001). L'abondance des juvéniles dans le milieu naturel, où ils ont longtemps été collectés, est un autre déterminant majeur dans le choix des espèces; c'est le cas notamment pour les cyprinidés parmi lesquels le silver barb (Barbodes gonionotus) s'est imposé dans la région. La valeur marchande est un autre critère de choix ; le sand goby, Oxyeleotris marmorata, est une espèce relativement fragile, mais dont la valeur marchande est la plus élevée des espèces d'eau douce, ce qui en fait une espèce d'intérêt aquacole.

\section{Bouleversements récents}

\section{Diminution des stocks sauvages}

Le contexte a changé rapidement dans le bassin du Mékong, depuis les années 1970 en Thailande, 1980 au Vietnam et 1990 au Cambodge et au Laos. La pression sur les milieux aquatiques s'est fortement accrue, directement ou indirectement, en raison de l'accroissement démographique et de l'essor des moyens de transport et des relations commerciales. La population totale du bassin du Mékong est estimée à 60 millions de personnes dont la moitié a moins de 15 ans (Baran, 2005). L'effort de pêche s'est accru de par l'augmentation du nombre de pêcheurs et la prise par unité d'effort (PUE), grâce à l'amélioration des techniques de pêche. Toujours d'après la même source, en 2000, le total des captures de poissons dans le Mékong était estimé à 880000 t. En 2002, la production totale (pêche et aquaculture) de produits animaux aquatiques (poissons, crustacés, mollusques) était estimée à 2033000 t. La consommation de produits aquatiques était alors supérieure à $30 \mathrm{~kg}$ par personne et par an dans le bassin du Mékong. Les statistiques officielles n'ont guère été actualisées. Une enquête effectuée récemment dans la zone transfrontalière entre le Laos et le Cambodge indique que la situation est alarmante (Cacot, 2007d). La zone en question couvre un tronçon de fleuve entre Paksé et Kratié long de $338 \mathrm{~km}$; cette zone est particulièrement importante pour la reproduction de nombreuses espèces de poissons du Mékong. Durant les dix dernières années, l'effort de pêche s'est accru de $273 \%$, alors que la quantité totale pêchée a baissé de $43 \%$; la prise par embarcation de pêche a chuté de $85 \%$.

Par ailleurs, de nombreux barrages ont été construits pour la production d'énergie ; au moins deux barrages sont opérationnels sur le cours principal du fleuve dans sa partie chinoise (sur les sites de Dawan et Dachaoshan); au moins sept autres barrages sont fonctionnels sur des affluents majeurs du fleuve; il reste, en fait, peu d'affluents qui ne soient pas encore perturbés. L'impact négatif de ces barrages sur les ressources aquatiques est bien documenté, comme pour celui de Dawan et celui des chutes de Yali (au Viêt-nam sur la rivière Sésan) (Montree, 2006), mais les constructions se poursuivent. Enfin, la majeure partie de la plaine d'inondation a été déboisée et utilisée pour la production agricole, l'intensification de l'agriculture s'accompagnant de l'utilisation de produits chimiques nocifs pour les organismes aquatiques. Au Cambodge, aux alentours du Ton Le Sap, il ne restait, en 2004, que $3 \%$ de la forêt inondable; sa surface est passée de 600000 à 19500 ha entre 1995 et 2004 (Baran, 2005).

Ces évolutions se sont traduites par la forte diminution des stocks naturels de poissons et surtout la raréfaction des espèces les plus grandes, dont l'âge à la première reproduction est de quatre à cinq ans. Aujourd'hui, la pêche est dominée par de petits cyprinidés dont la reproduction est précoce et la fécondité élevée; Henicorbynchus sp., Barbodes sp. et Morulius krysophekadion sont les espèces les plus pêchées actuellement.

\section{Concurrence des espèces exotiques}

Avec la raréfaction des juvéniles sauvages, les pisciculteurs ont porté leurs efforts sur le contrôle de la reproduction avec des géniteurs maintenus en captivité. Cela n'a, cependant, concerné qu'un nombre relativement restreint d'espèces; aujourd'hui $B$. gonionotus et $P$. bypophthalmus dominent la production d'espèces indigènes dans la région. Cette situation est liée en particulier à la disponibilité en fingerlings (poissons de la taille d'un doigt, c'est-à-dire allant de 5 
à $15 \mathrm{~g}$ ) d'espèces exotiques introduites, notamment, pour la pisciculture depuis les années 1970 ; au total 17 espèces ont été introduites (Welcomme et Vidthayanon, 2003). Comme partout ailleurs, les introductions d'espèces sont destinées à améliorer l'aquaculture et la pêche (Lazard et Levêque, 2009). Les espèces exotiques les plus répandues dans la région sont la carpe commune, le tilapia et le poisson-chat africain, Clarias gariepinus ; plusieurs espèces de carpes chinoises et de carpes indiennes sont également bien représentées. Les statistiques de la FAO pour 2005 (FIGIS, 2005) indiquent que la production d'espèces exotiques est globalement de $14 \%$ pour les quatre pays riverains du Mékong (tableau 1), sur une production aquacole en eau douce estimée à 1,35 million de tonnes. La part des espèces exotiques est certainement plus élevée (30-40\%?), mais elle n'est pas déterminée pour le Vietnam. Néanmoins, les espèces indigènes résistent avec une part globale de $40 \%$. Le reste est constitué d'espèces "indéterminées ".

La production d'espèces exotiques aggrave encore la raréfaction des espèces indigènes en raison de la concurrence qu'elles exercent au niveau des niches écologiques. Des fingerlings sont, en effet, relâchés en permanence dans les lacs de barrage (Bartley et al., 2005). De plus, une quantité non négligeable de juvéniles s'échappe des écloseries, et des poissons de toutes tailles se retrouvent aussi dans le fleuve lorsqu'une forte crue submerge les digues des étangs. Par conséquent, plusieurs espèces exotiques sont, aujourd'hui, relativement abondantes dans le fleuve; neuf espèces y sont même bien établies incluant Cirrbinus cirrhosus, C. gariepinus, Gambusia affinis, Helostoma temminki, Labeo robita, Oreochromis mossambicus, Oreochromis nilo- ticus, Poecilia reticulata, Pterygoplichthys plecostomus (Welcomme et Vidthayanom, 2003). À l'extrême sud du Laos, la carpe commune représente le tiers des captures (Cacot, 2007d).

\section{Menace hybride}

La pisciculture affecte également la biodiversité par la production d'hybrides obtenus avec des espèces indigènes. L'hybridation concerne deux espèces de poissons-chats: Clarias macrocephalus et $P$. hypophthalmus. Des femelles de C. macrocephalus sont croisées avec des mâles de C. gariepinus (espèce africaine); le croisement est effectué pour "améliorer" la croissance de l'espèce locale (espèce naine). Les femelles de $P$. hypophthalmus sont croisées avec les mâles de plusieurs autres espèces indigènes de Pangasiidae dont Pangasianodon gigas, $P$. bocourti et Pangasius larnaudii. L'hybridation de $P$. bypophthalmus est effectuée par commodité, car l'obtention des ovules des autres pangasidés n'est pas aisée. Elle permet également d'améliorer l'image du "produit ", sachant que le pur $P$. hypophthalmus est un poisson diversement apprécié. Les hybrides de Clarias sont produits massivement au Vietnam et en Thailande ; les hybrides de Pangasiidae sont produits en Thailande et au Laos. L'hybridation constitue une menace pour le patrimoine génétique des espèces indigènes, car les hybrides produits ne sont pas stériles; l'impact est déjà net en Thaillande où les purs C. macrocephalus sont devenus très rares (Senanan et al., 2004). La fertilité de l'hybride entre $P$. bypophthalmus (femelle) et $P$. bocourti (mâle) a été récemment démontrée (Campet et al., 2008).
La domestication aujourd'hui

\section{Pour la conservation de la biodiversité}

Les actions récentes ou en cours concernent l'optimisation du cycle d'élevage des espèces indigènes déjà bien connues et la maitrise de la reproduction en captivité de " nouvelles" espèces d'intérêt aquacole. Ces dernières sont pour la plupart devenues rares dans le milieu naturel; la domestication vient alors au secours de la biodiversité. Trois espèces " géantes " sont particulièrement emblématiques des efforts entrepris : le poisson-chat $P$. gigas et les carpes Catlocarpio siamensis et Probarbus julieni; leur poids vif atteint 300, 200 et $60 \mathrm{~kg}$, respectivement (Mattson et al., 2002). Deux grands migrateurs anadromes du fleuve, Pangasius krempfi et $P$. mekongensis, font également l'objet d'une attention particulière. Enfin, les poissons les plus chers sont aussi étudiés, comme les deux poissons-chats Hemibagrus wyckioides et Micronema sp. Enfin, le cyprinidé Cirrbinus microlepis fait également l'objet de travaux de domestication.

Les travaux sont conduits par les instituts de recherche nationaux des pays riverains du Mékong et le MRC qui s'attache à fédérer les programmes nationaux (www. mrcmekong.org). L'approche se veut donc moderne, scientifique, en rupture avec les tests empiriques effectués jusqu'alors par les pisciculteurs euxmêmes. C'est dans ce contexte que s'intègre le projet du Cirad mené avec les partenaires locaux depuis 1994 au Vietnam (jusqu'en 2005), puis au Laos. Quatre espèces sont particulièrement étudiées dans le cadre du projet conduit actuelle-

\section{Tableau 1. La production aquacole des poissons d'eau douce en fonction de leur origine dans les pays riverains du Bas-Mékong (d'après les statistiques de la FAO en 2005).}

Table 1. Production from aquaculture of freshwater fish in the Lower Mekong River Basin according to the origin of the fish species (2005 FAO statistics).

\begin{tabular}{llllll}
\hline Origine des espèces & \multicolumn{1}{c}{ Cambodge } & \multicolumn{1}{c}{ Laos } & \multicolumn{1}{c}{ Thaïlande } & \multicolumn{1}{c}{ Vietnam } & \multicolumn{1}{c}{ Total } \\
\hline Indigène & $17900(70 \%)$ & $15040(19 \%)$ & $136232(37 \%)$ & $376000(43 \%)$ & $545172(40 \%)$ \\
Exotique & $7600(30 \%)$ & $62960(81 \%)$ & $120209(32 \%)$ & - & $190769(14 \%)$ \\
Hybride & - & - & - & $114311(31 \%)$ & - \\
Indéterminée & - & 78000 & - & $501100(57 \%)$ & $501100(37 \%)$ \\
Total & 25500 & 370752 & 877100 & 1351352 \\
\hline
\end{tabular}


ment au Laos: H. wyckioides, P. krempfi, C. microlepis et Probarbus jullieni (figure 3) (Cacot, 2004, 2005, 2007a, 2007b, 2007c).

\section{Enjeux économiques}

Les enjeux de la domestication sont tangibles au niveau économique, dans un contexte où la pisciculture asiatique est en pleine "mondialisation". D'une manière générale, les poissons indigènes sont nettement plus appréciés que les poissons exotiques par les consommateurs locaux; les prix de vente moyens au Laos sont respectivement de 2,4 et de $1,2 \mathrm{US} \$ / \mathrm{kg}$, (figure 4). La pisciculture permet également de commercialiser des poissons en dehors des périodes de pêche abondante. Enfin, plusieurs espè-
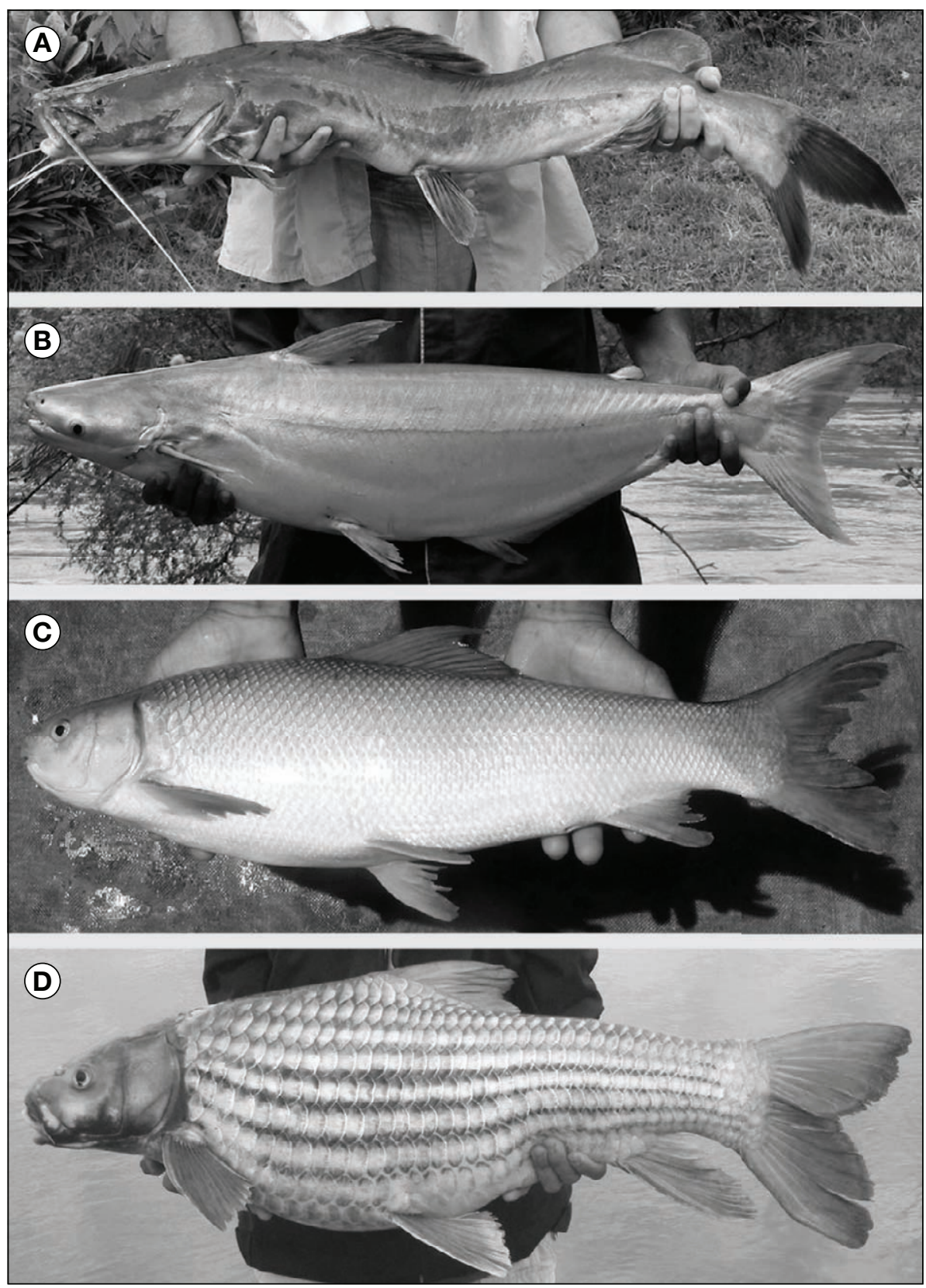

Figure 3. Les espèces étudiées pour la domestication dans le cadre du programme de recherche mené par le Cirad et les partenaires locaux au Laos.

Figure 3. The fish species for the domestication in the frame of the research programme conducted by the Cirad with local partners in Laos.

A) Hemibagrus wyckioides ; B) Pangasius krempfi ; C) Cirrhinus microlepis ; D) Probarbus jullieni.

ces indigènes présentent un potentiel aquacole considérable qui peut même dépasser les performances des espèces exotiques.

Le cas de $P$. hypophthalmus est particulièrement intéressant. Cette espèce indigène cumule des avantages de façon inégalée parmi toutes les espèces connues en pisciculture. Ainsi, P. bypophthalmus présente une grande tolérance vis-à-vis de la qualité de l'eau (respiration aérienne), une croissance rapide (1 kg en huit mois), un régime alimentaire omnivore à tendance végétarienne (besoins restreints en protéines), une chair de qualité (absence d'arêtes intramusculaires) et une fécondité très élevée (jusqu'à 200000 œufs par kilogramme de poids vif et par ponte). Ce poisson vit, en effet, dans la plaine d'inondation du Mékong pour se nourrir de fruits, et il peut supporter l'eau faiblement oxygénée des mares et des bras de rivière stagnants. Aujourd'hui, P. hypophthalmus est produit dans le delta du Mékong en étangs avec des rendements compris entre 100 et $600 \mathrm{t} / \mathrm{ha}$ par an en fonction du renouvellement de l'eau ; son alimentation repose sur le son de riz produit localement (60\% en poids des ingrédients de l'aliment qu'il consomme); le principal débouché est l'exportation de filets congelés. La production a connu un essor exceptionnel entre 1995 et 2007, passant de 15000 tonnes à un million de tonnes ; le chiffre d'affaire de cette filière avoisine le milliard de dollars US. P. hypophthalmus surpasse donc en termes de production les autres poissons-chats d'Amérique du Nord (Ictalurus punctatus), d'Afrique ( $C$. gariepinus) et d'Europe (Silurus glanis). Il est intéressant de rappeler que I. punctatus avait été envisagé dans les années 1990 comme espèce de substitution aux pangasidés du delta, lorsque leur reproduction artificielle n'était pas encore maitrisée.

Plusieurs autres espèces indigènes du Mékong réservent également un potentiel intéressant. C'est le cas en particulier de deux autres Pangasiidae, P. krempfi et $P$. mekongensis, qui vivent naturellement dans l'estuaire du fleuve et qui seraient donc bien adaptés à la pisciculture en eau saumâtre; dans ce milieu, le choix des espèces de poissons d'intérêt aquacole avéré est relativement restreint. Par ailleurs, en eau douce, la domestication de la "carpe " géante et planctophage C. siamensis contribuera à l'amélioration du rendement de la pisciculture en étangs fertilisés, de même que les "carpes" C. microlepis et $P$. julieni. 


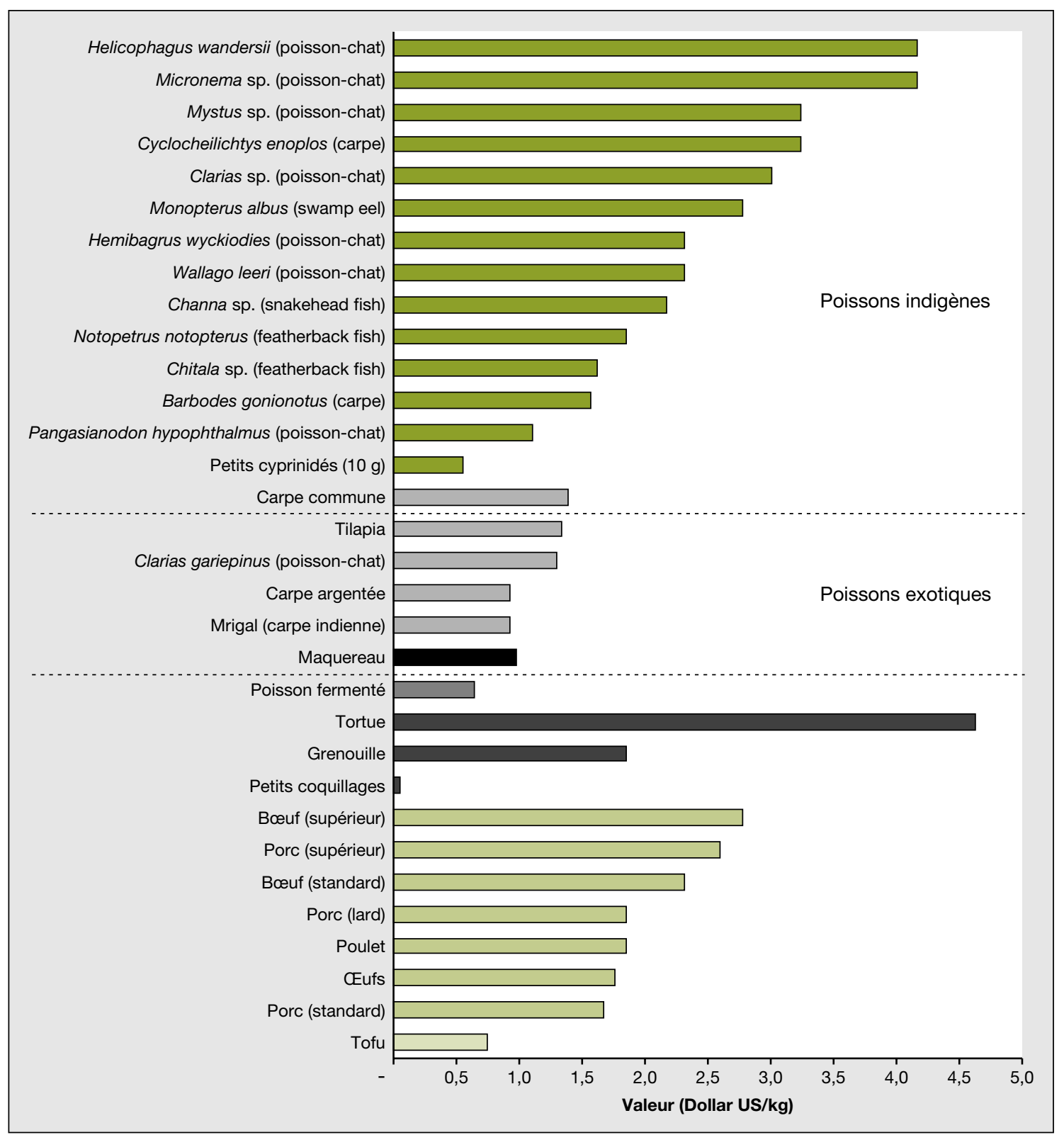

Figure 4. Prix des poissons et des autres sources de protéines alimentaires au Laos sur le marché de détail à Savannaketh (Laos) en novembre 2005.

Figure 4. Price per fish species and other sources of food protein in Laos on the retail market in the city of Savannaketh (Laos PDR) in November 2005.

\section{Typologie}

\section{des espèces indigènes d'intérêt aquacole}

\section{Présentation générale}

Aujourd'hui 32 espèces indigènes du Mékong sont élevées en pisciculture dans le bassin du Mékong. Le choix de ces espèces s'articule autour de cinq critères principaux qui sont la reproduction, la tolérance vis-à-vis de la qualité de l'eau, la croissance, l'alimentation et la valeur marchande (figure 5). Le tableau 2 récapitule les performances relatives de ces espèces ainsi que l'ordre de grandeur de leur production; les performances sont illustrées dans la figure 6 pour les quatre principales espèces produites. Les ordres de grandeur de production sont issus de la seule connaissance personnelle des auteurs, la production exacte de chaque espèce n'étant pas connue. Les dernières statistiques globales font état de 260000 t de production aquacole d'espèces indigènes pour l'ensemble de la région du BasMékong (Phillips, 2002). On peut, cependant, remarquer que le niveau de produc- 


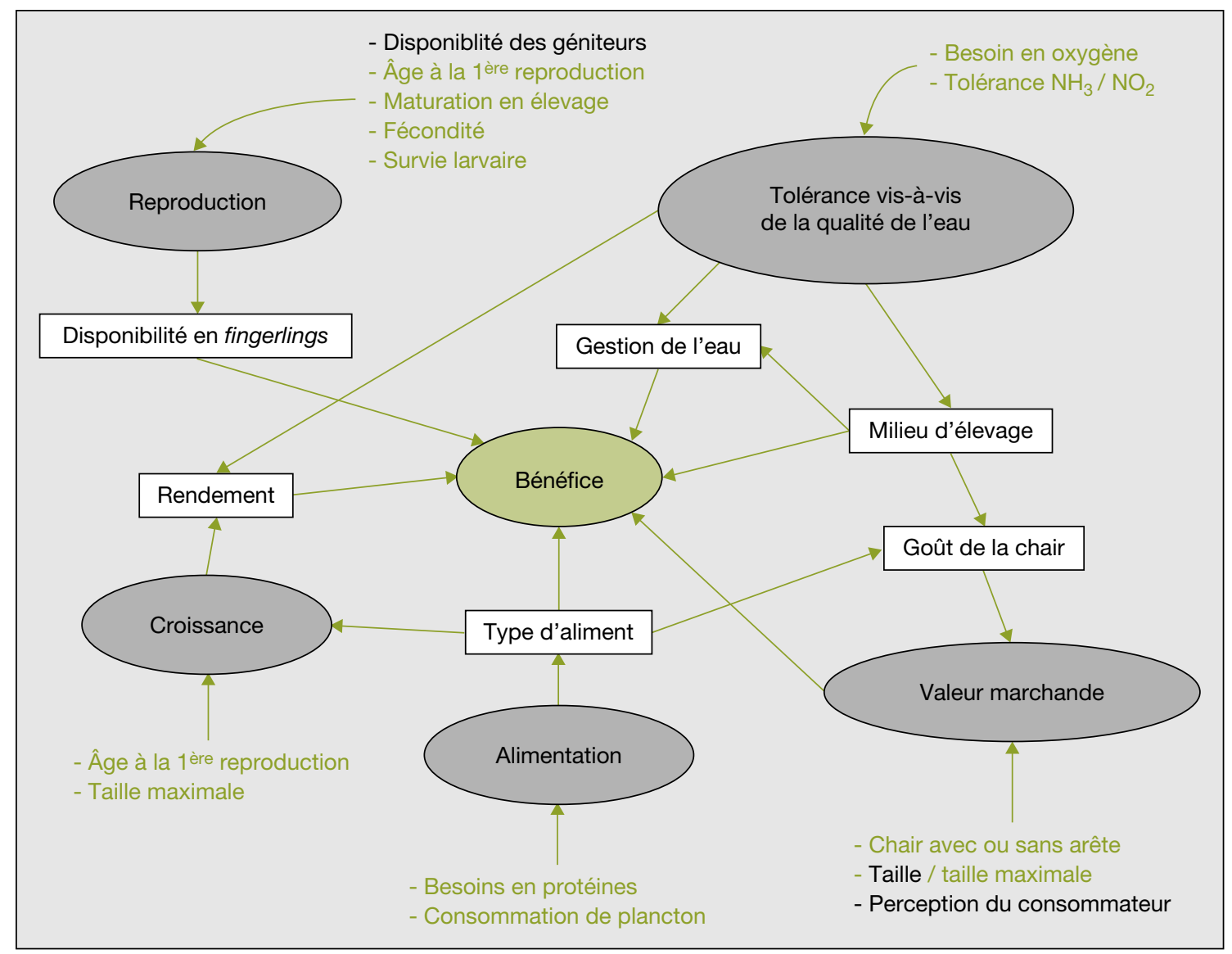

Figure 5. Principaux éléments déterminants du choix des espèces parmi les poissons indigènes du Mékong.

Figure 5. Main factors for the choice of the indigenous Mekong fish species.

tion est très variable selon ces espèces ; $P$. hypophthalmus est l'espèce largement dominante avec $\times 100000 \mathrm{t}$ par an, suivie de trois autres espèces $(\times 10000 t)$ et de huit autres de moindre importance $(\times 1000 \mathrm{t})$; les 20 autres espèces sont produites à des niveaux relativement faibles. À ces espèces indigènes pures s'ajoutent des hybrides produits avec C. macrocephalus $(\times 10000$ t) et P. hypophthalmus $(\times 1000 \mathrm{t})$.

Enfin, il y a une certaine interdépendance entre le choix des espèces, d'une part, et le type de système de production et son niveau d'intensification, d'autre part. Ainsi, le type d'alimentation et le niveau de rusticité des espèces constituent deux éléments déterminants majeurs, comme illustré dans la figure 7 , avec un panel d'espèces représentatif. La plupart des espèces convient généralement à deux, voire trois, systèmes de production différents.

\section{Potentiel aquacole en question}

Un critère synthétique définit le " potentiel aquacole " qui a été calculé en combinant les cinq critères de performance dans l'équation linéaire suivante : potentiel $=0,736$ reproduction $+0,317$ rusticité $+0,251$ valeur marchande-0,091 croissance $-0,226$ alimentation ; la valeur de chacun des cinq paramètres est ajustée entre 1 et 5 . Ce modèle s'avère pertinent, car il rend compte de $71 \%$ de la variabilité des niveaux de production (figure 8). La valeur des coefficients affectée à chaque paramètre indique que la reproduction est le principal élément déterminant $(+100 \%)$, suivi de la rusticité (+43\%) et de la valeur marchande $(+34 \%)$. De façon surprenante, le paramètre " alimentation des poissons" (inverse des besoins en protéines) conditionne négativement le potentiel aquacole $(-31 \%)$. Enfin, la croissance a une faible incidence, et elle est même négative ( $-12 \%)$. Cette modélisation reflète la production importante de l'espèce carnassière C. micropeltes et de trois espèces de petite taille au régime alimentaire omnivore à tendance carnassière (Anabas testudineus, Clarias sp., Trichogaster pectoralis). Ces quatre espèces sont à la fois très rustiques et appréciées des consommateurs locaux. Elles sont bien adaptées à l'environnement de la plaine d'inondation; leur respiration aérienne complémentaire leur permet, en effet, de survivre dans des eaux faiblement oxygénées durant la saison sèche. L'alimentation de C. micropeltes 


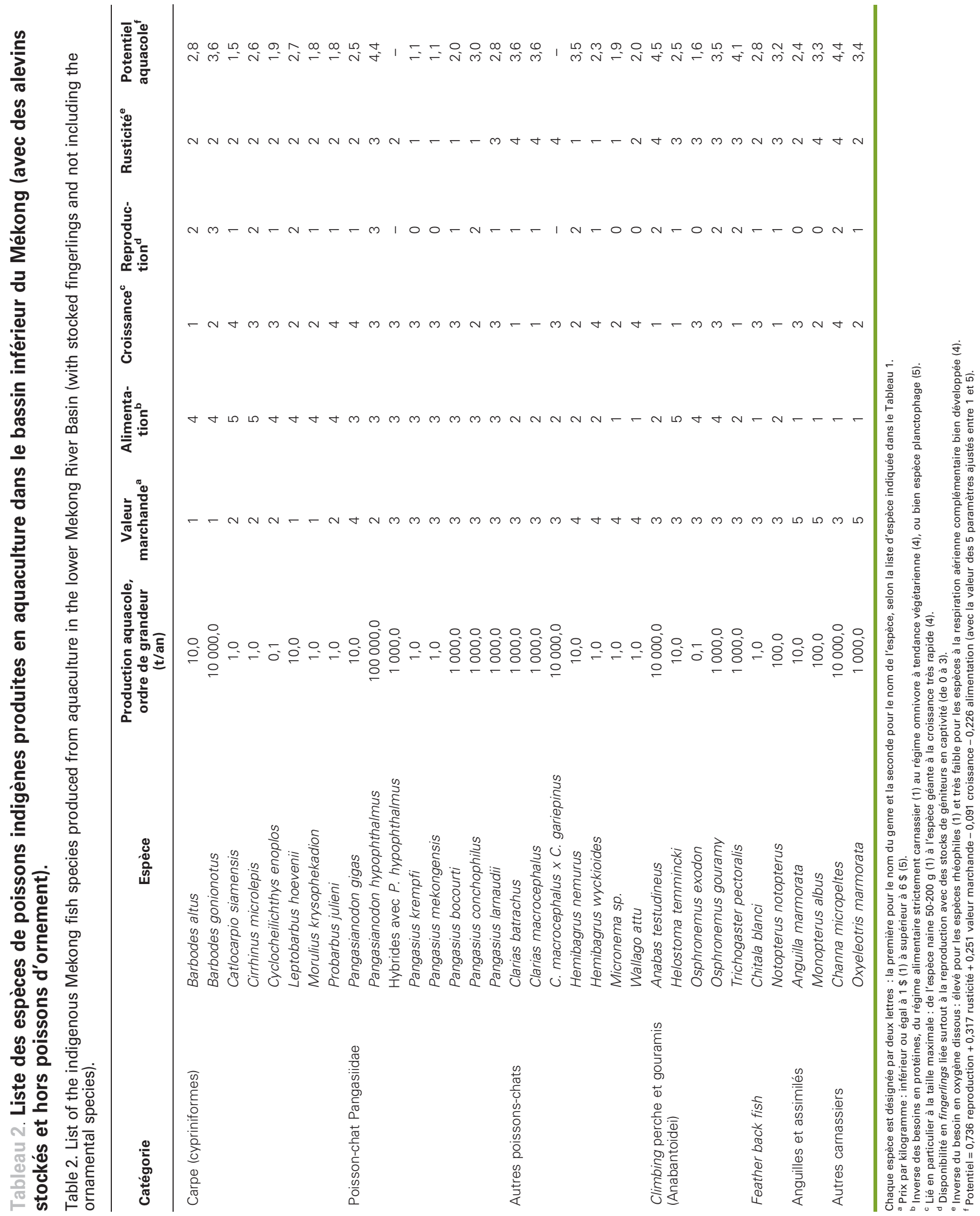




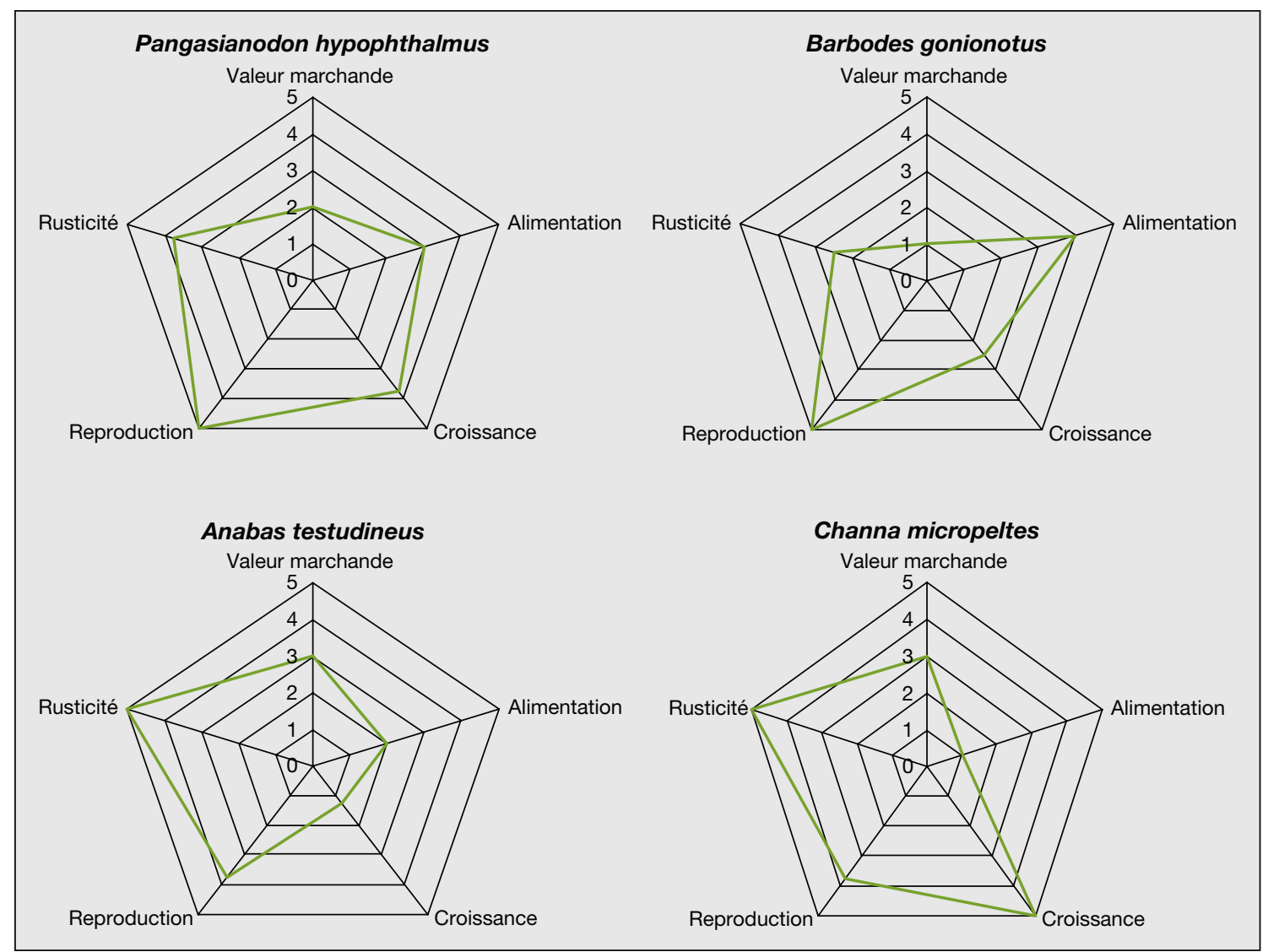

Figure 6. Potentiel aquacole comparé des quatre espèces de poissons indigènes les plus produites dans le bassin du Mékong.

Figure 6. Comparison of the aquaculture potential of the four major fish species produced from aquaculture in the lower Mekong River Basin.

repose beaucoup sur la valorisation du "poisson fourrage ". Les trois espèces de plus petite taille sont quant à elles très répandues pour la rizipisciculture; elles se nourrissent en particulier des insectes ravageurs du riz.

\section{Conclusion}

La place des espèces indigènes du Mékong dans l'aquaculture locale évolue sous l'influence de divers atouts et contraintes. Ainsi, la ressource en protéines pour l'alimentation des poissons devient un facteur limitant majeur. La disponibilité en poisson fourrage diminue rapidement (Suchart et al., 2005), et son exploitation est même volontairement restreinte au Cambodge pour préserver la ressource. Dans ce pays, l'élevage de C. micropeltes (espèce carnassière) a été officiellement interdit depuis 2006 (Edwards, 2008) ; son remplacement par des espèces omnivores est encouragé, mais dans les faits la transition n'est pas aisée ; les fingerlings ne sont pas disponibles en quantités suffisantes de même que les sources alternatives de protéines alimentaires.

Le processus de domestication repose essentiellement sur le dynamisme des pisciculteurs parmi les plus innovants ainsi que sur les recherches conduites par les organismes de R\&D. La communication entre tous ces acteurs constitue un élément déterminant pour favoriser les transferts de technologie, au niveau de chaque pays et à l'échelle régionale. Les avancées sur telle ou telle espèce ou discipline sont, en effet, très variables d'une région à l'autre. Par ailleurs, les organismes de R\&D doivent œuvrer en faveur de la préserva- tion des populations sauvages de poissons. La diversité ichtyologique est, en effet, indispensable à la domestication, tant au niveau du choix des espèces que du maintien de la diversité génétique au sein de chaque espèce.

Certaines espèces indigènes du Mékong deviennent des espèces exotiques dans plusieurs régions du monde où elles sont introduites. C'est le cas principalement de P. bypophthalmus dont l'introduction dans l'archipel indonésien remonte aux années 1970 (Legendre, 1999). Aujourd'hui, cette espèce est présente dans tous les pays d'Asie du Sud et du Sud-Est ainsi qu'en Chine. Des introductions sont à craindre en Amérique du Sud et en Afrique. Dans ces régions, il faut s'attendre à ce que $P$. hypophthalmus affecte les populations indigènes de poissons, car il s'agit d'une espèce rustique et prolifique (mais non carnassière). 


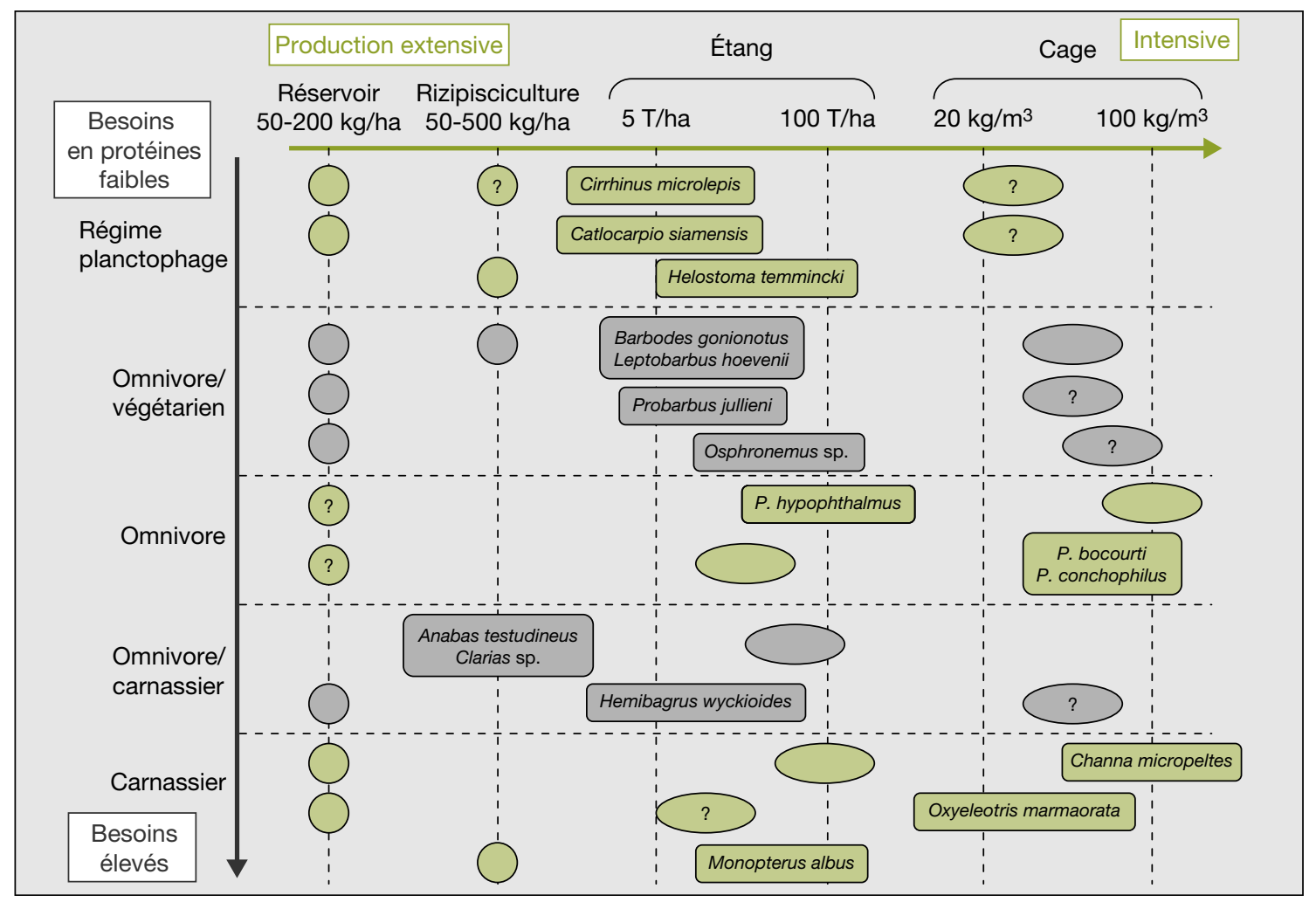

Figure 7. Répartition d'espèces indigènes dans les systèmes de production en fonction du niveau d'intensification et du régime alimentaire.

Figure 7. Various shares of the indigenous Mekong fish species among the production systems according to the level of intensification and the feeding regime.

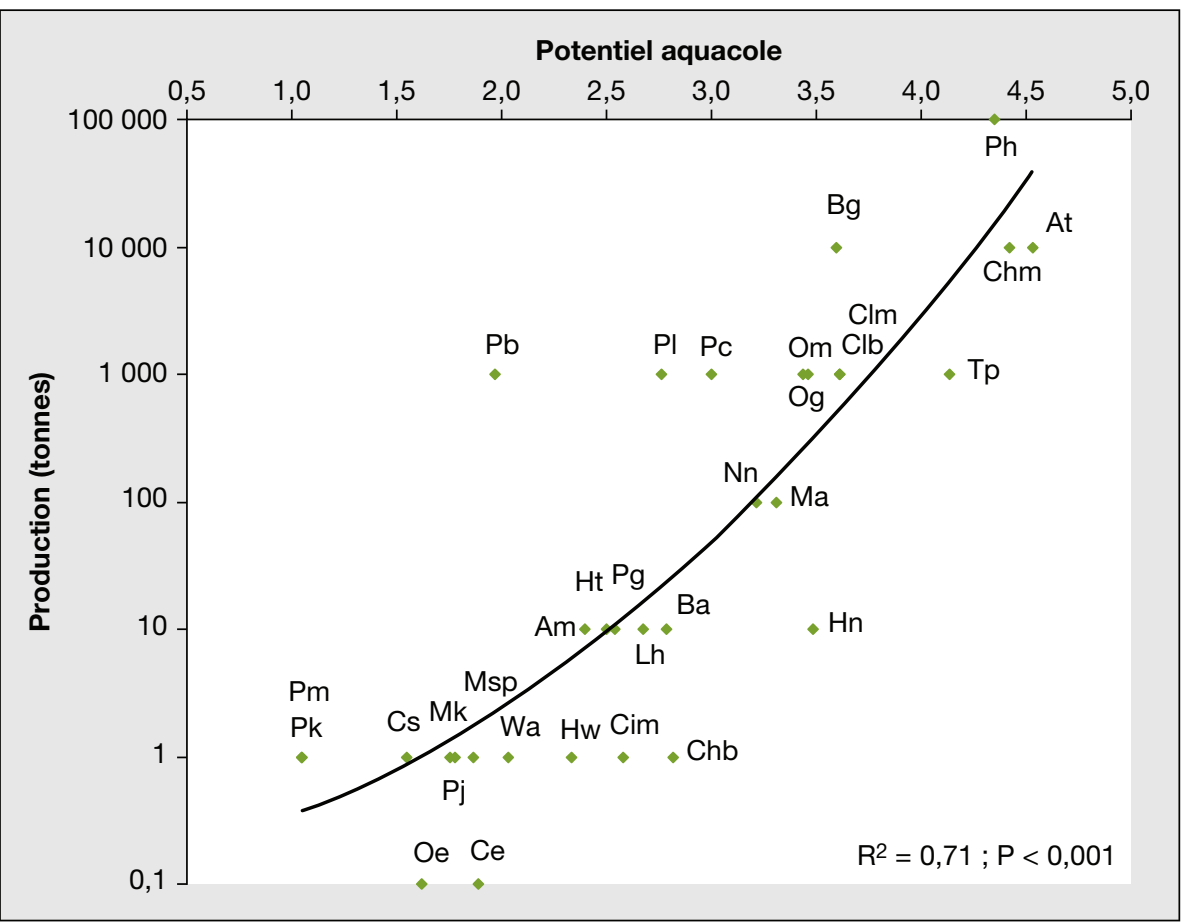

Figure 8. Relation entre la production actuelle (ordre de grandeur) et le potentiel aquacole des 32 espèces de poissons indigènes produits dans le bassin du Mékong.

Figure 8. Relationship between the ongoing production (order of magnitude) and aquaculture potential of the 32 indigenous fish species produced in the lower Mekong River Basin.

\section{Références}

Baran E. Cambodia inland fisheries: facts, figures and context. Phnom Penh (Cambodge): WorldFish Center and Inland Fisheries Research and Development Institute, 2005.

Bartley DM, Bhujel RC, Funge-Smith S, Olin PG, Phillips MJ. International mechanisms for the control and responsible use of alien species in aquatic ecosystems. In: Report of an ad hoc expert consultation. Xishuangbanna, people's Republic of China, 27-30 August 2003. Rome (Italie): FAO, 2005.

Cacot P. Présentation de la pisciculture en cages flottantes dans le Sud Vietnam. Caractéristiques de l'élevage sur le Mékong de Pangasius pangasius. Montpellier (France): CiradEMVT ; GAMET, 1993.

Cacot P. Domestication of the indigenous Mekong catfish Pangasius krempfi: overview of the fishery in Cambodia and Laos and preliminary study of the artificial reproduction above the Khone waterfalls. Activity report. Montpellier (France) : Cirad-Aquaculture Unit; MRC, 2004.

Cacot P. Survey at the Khone waterfalls and experiments on the propagation of Pa-phone (Cirrhinus microlepis) carried out at the $\mathrm{km} 8$ station (Pakse). Activity Report 2005. Vientiane (Laos): Cirad-Aquaculture Unit ; NAFRILARReC, 2005. 
Cacot P. Advances in the reproduction of several Mekong fish species performed with wild and captive fish breeders in the Champasak Province. Activity report 2006. Vientiane (Laos): Cirad-Aquaculture Unit ; NAFRILARReC, 2007.

Cacot $\mathrm{P}$. Control of the reproduction and the nursing of Pa-kheung (Hemibagrus wyckioides) and advances with three other species. Domestication of the indigenous fish species in Laos. Activity report for the breeding season 2007. Vientiane (Laos): Cirad-Aquaculture Unit ; NAFRI-LARReC project, 2007.

Cacot P. Trial on the reproduction of the carp Pa-eun (Probarbus jullieni) with fish breeders collected from the Mekong river at the Don Kho Island (Dec. 07). Activity report. Vientiane (Laos): Cirad-Aquaculture Unit ; NAFRILARReC project, 2007.

Cacot P. Preliminary study for the LaoCambodia transboundary project. Consultant report. Vientiane (Laos): Cirad-Aquaculture Unit ; FAO, 2007.

Campet M, Nguyen Hoang Vu, Cacot P, Lazard J. Comparative study of aquaculture potential of two Pangasiidae species, Pangasius bocourti, Pangasianodon hypophthalmus and their hybrid ( $P$. hypophthalmus $\times$ P. bocourti). In: The proceedings of the WAS symposium in Busan, South Korea, May 19-23, 2008. Baton Rouge (USA): World Aquaculture Society, 2008. (in press).
Chevey P Le Poulain F. La pêche dans les eaux douces du Cambodge. Nha Trang (Vietnam) Institut océanographique de I'Indochine, consultable à I'Institut océanographique de Monaco, 1940.

Edwards P. Peter Edwards writes on rura aquaculture: Cambodian Government ban on snakehead farming enforce. 2008. http:// library.enaca.org/AquacultureAsia/Articles/ jan-march-2008/edwards-jan-08.pdf

FIGIS (Fisheries Global Information System). www.fao.org/figis/servlet/), 2005.

Guibert R. La pangasiculture dans le delta du Mékong (Vietnam): suivi de la qualité de l'eau en étangs et évaluation de la qualité des produits. Thèse de master, INAPGAgroParisTech, département des productions animales, 2003.

Huillery AL. Étude de la filière de production de poissons-chats Pangasiidae dans le delta du Mékong (sud du Vietnam). Thèse de master, INAPGAgroParisTech, département des productions animales, 2001.

Lazard J, Lévêque C. Introductions et transferts d'espèces de poissons d'eau douce. Cah Agric 2009 ; 18 : 157-63. Doi : 10.1684/agr.2009.0290

Legendre M. The catfish Asia project: back grounds, aims and prospects. In: Legendre M, Pariselle A, eds. The biological diversity and aquaculture of Clariid and Pangasiid Catfish in Southeast Asia. Proc. mid-term works hop of the "Catfish Asia project". Paris: IRD, 1999.
Mattson NS, Buakhamvongsa K, Sukumasavin N, Tuan N, Vibol O. Cambodia Mekong giant fish species: on their management and biology. MRC Technical Paper $\mathrm{n}^{\circ} 3$. Phnom Penh (Cambodge): Mekong River Commission, 2002.

Montree C. The Mekong's changing currency. Watershed 2006; 11 : 12-25.

Phillips MJ. Fresh water aquaculture in the lower Mekong basin. MRC Technical Paper $\mathrm{n}^{\circ}$ 7. Phnom Penh (Cambdoge): Mekong River Commission, 2002.

Rainboth WJ. Species identification field guide for fishery purposes: fishes of the Cambodian Mekong. Rome (Italie): FAO, 1996.

Senanan W, Kapuscinski AR, Na-Nakorn U Miller LM. Genetic impacts of hybrid catfish farming (Clarias macrocephalus $\times$ C. gariepinus) on native catfish populations in central Thailand. Aquaculture 2004 ; 235 : 167-84.

Suchart I, Niklas SM, Kent GH. Use of inland trash fish for aquaculture feed in the lower Mekong basin in Thailand and Lao PDR. Proceedings of the "Regional workshop on the low value and "trash fish" in the Asia-Pacific region" Hanoi, Vietnam, 7-9 June 2005.

Welcomme RL. River Fisheries. Fish Tech Pap n²62. Rome (Italie): FAO, 1985.

Welcomme RL, Vidthayanon C. The impacts of introductions and stocking of exotic species in the Mekong basin and policies for their control. MRC Technical Paper $n^{\circ}$ 9. Phnom Penh : Mekong River Commission, 2003. 